\title{
La región de Liberec como destino turístico: un análisis de caso en la República Checa*
}

\author{
Gabriela Antošová** \\ Helmuth Yesid Arias Gómez*** \\ Recibido: 2013-10-02 Aprobado: 2013-12-15 Disponible en línea: 2014-05-30 \\ doi:I0.III44/Javeriana.CDRII-73.rldt
}

Cómo citar este artículo: Antošová, G. y Arias-Gómez, H. Y. (20I4). La región de Liberec como destino turístico: un análisis de caso en la República Checa. Cuadernos de desarrollo rural, II(73), 39-59. doi:10.III44/Javeriana. CDRII-73.rldt

\section{Resumen}

El artículo pretende, a partir de alguna conceptualización teórica, hacer una descripción de Liberec como destino turístico, una región relativamente atrasada de la República Checa. Para el análisis propuesto se aplicó un enfoque multidisciplinario, basado en las metodologías de multiplicadores y de encuestas a los visitantes, alcaldes y habitantes, las cuales proporcionaron información acerca de la ejecución de proyectos de desarrollo financiados por la Unión Europea y la percepción de los agentes locales con respecto a estos. Se corroboró el papel del turismo en el desarrollo económico de la región y la necesidad de potenciar los atractivos turísticos de Liberec en el marco de una explotación económica sustentable, que haga partícipe a los agentes locales de los beneficios del turismo cultural rural.

\section{Palabras clave autores:}

destino rural; turismo rural y cultural; desarrollo rural y cultural; región de Liberec

\section{Palabras clave descriptores:}

turismo rural; turismo cultural; desarrollo rural; desarrollo cultural; Liberec (República Checa)

\footnotetext{
*Este artículo de investigación ha sido elaborado gracias al esquema de ayudas con código IGA 20121054:IIIIO/1312/3112, "la explotación financiera de los fondos para el desarrollo rural en la Unión Europea”, suministradas por la Agencia Interna de Ayudas de la Facultad de Economía y Dirección de Empresas de la Universidad Agrícola Checa, de Praga (República Checa).

**Estudiante de Doctorado, Universidad Agrícola Checa, departamento de Humanidades, Praga, República Checa. Correo electrónico: antosovag@pef.czu.cz

***Estudiante de Doctorado, Análisis Económico Aplicado e Historia Económica. Universidad de Sevilla, España. Máster en Ciencias Económicas Universidad Nacional de Colombia. Correo electrónico: hyarias@gmail.com
} 


\title{
The Liberec Region as a Tourism Destination: a Case Study in the Czech Republic
}

\begin{abstract}
From a theoretical conceptualization the article seeks to make a description of Liberec, a relatively backward region of the Czech Republic. For the proposed analysis a multidisciplinary approach was applied. It was based on the multidisciplinary approaches and the surveys taken by visitors, mayors and citizens. These provided information about the implementation of development projects financed by the European Union and the perception of local agents. It was confirmed the role of tourism in the economic development of the region and the need to enhance Liberec's tourist attractions in the context of a sustainable economic exploitation that must share with the local agents the benefits of rural cultural tourism.
\end{abstract}

Keywords author:

rural destination; rural and cultural tourism; rural and cultural development; Liberec region.

Keywords plus:

rural tourism; cultural tourism; rural development; cultural development; Liberec (Czech Republic).

\section{La région de Liberec comme destination touristique: une étude de cas en République Tchèque}

\section{Résumé}

L'article vise, à partir de quelque conceptualisation théorique, à faire une description de Liberec comme destination touristique, une région relativement sous-développée de la République Tchèque. Pour l'analyse proposée, il a été recommandé une approche multidisciplinaire, basée sur les méthodes de multiplicateurs et d'enquêtes aux visiteurs, aux maires et aux habitants; ces méthodes-ci ont fourni des informations à propos de la mise en marche des projets de développement financés par l’Union Européenne et la perception des agents locaux sur ce sujet. Le rôle du tourisme dans le développement économique de la région et le besoin de potentialiser les aspects touristiques positifs de Liberec ont été confirmés sous le cadre d'une exploitation économique durable qui fasse participer les agents locaux des bénéfices du tourisme cultural rural.

\section{Mots-clés auteur:}

destination rurale; tourisme rural et culturel; développement rural et culturel; région de Liberec.

\section{Mots-clés descripteur:}

tourisme rural; tourisme culturel; développement rural; développement culturel; Liberec (République Tchèque). 


\section{Introducción}

El concepto "destino rural" como "un atractor" de turismo y generador de desarrollo económico integra muchos elementos: importantes accesos y puertas de entrada, comunidades, complejos de atracción y corredores de comunicación (Gunn, 2002). Idealmente, estos elementos necesitan ser integrados al turismo cultural rural para ser exitosos. El turismo rural se conoce también como "turismo cultural rural" (MacDonald y Jolliffe, 2003). Los teóricos del turismo (Pearce, 198I; Garrod, Wornell y Youell, 2006) por lo general coinciden en que un área de destino turístico requiere muchos componentes esenciales.

Esos componentes incluyen: una atracción focal, una herencia cultural, servicios de sanidad, complementos a las atracciones focales, actividades suplementarias, sistemas de acceso, una comunidad y características de integración. También se incluye el ambiente completo y el desarrollo del turismo a cierta escala en un área y el análisis de la forma en que la actividad esté bien integrada en la vida social y económica de la comunidad. Muchos autores (Feiner, Mi y Schmid, 2002; Kausar y Nishikawa, 2010) han identificado los impactos tanto positivos como negativos del desarrollo rural y cultural, sus éxitos y sus fracasos, en particular, la forma como este es planeado y gestionado, y más importante aun, cómo lo asumen los agentes, incluida la comunidad (Ziakas y Costa, 20Io; Panis, 2009) y cómo están ellos involucrados en la planificación y en el proceso de desarrollo (Niezgoda y Zmyślony, 2003).

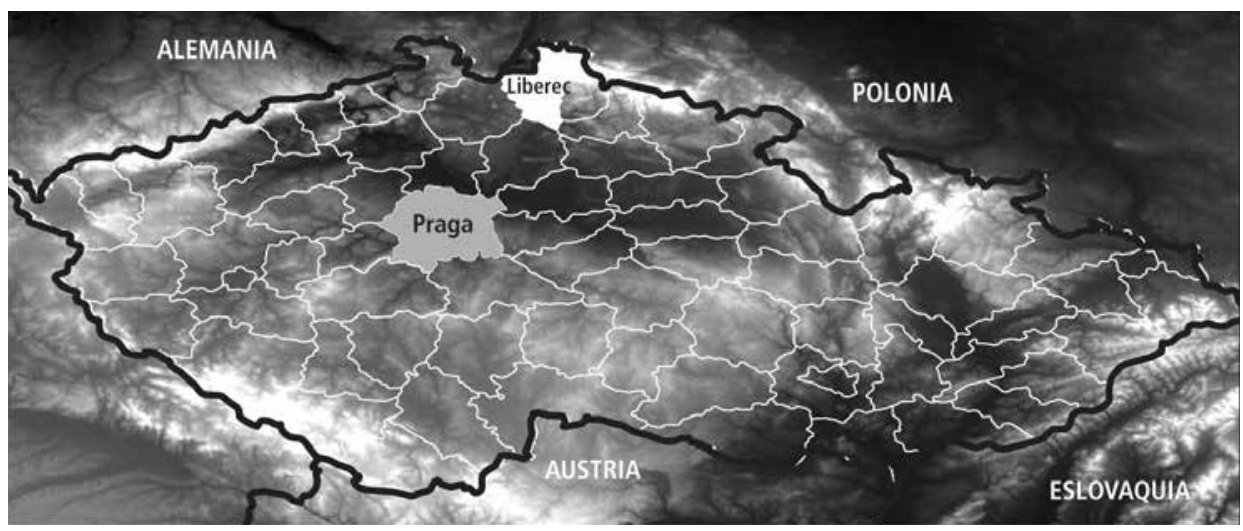

Figura i. Detalle del distrito Liberec en la República Checa FUENTE: elaboración propia con archivos shape de Diva-Gis.org 
Otros han establecido nociones de turismo alternativo (Kürker y Dinçyürek, 2007) que podrían ser consideradas como formas más livianas de turismo que los enfoques tradicionales dominantes (Porto, Leanza y Cascone, 20I2) sobre desarrollo cultural rural, por ejemplo: el turismo rural. Este tipo de turismo está promocionado por sus impactos positivos (Mason, 2003), las oportunidades de empleo, la preservación de la herencia cultural y los mercados culturales, la protección de los monumentos, la recuperación de aspectos culturales perdidos y amenazados, tales como artesanías, lenguas, etc. Por otro lado, se pueden reconocer sus impactos negativos como congestión, suciedad y deterioro ambiental en los destinos, etc. Otra importante corriente en la investigación en turismo cultural rural se concentró en el impacto económico de los monumentos de herencia y los megaeventos (Skoczek, 2003).

Los resultados previos de la investigación (Antošová, 2013a) fueron usados para la siguiente etapa del proyecto sobre desarrollo de turismo rural en la región de Liberec. Antes de la etapa de análisis se habían escogido en profundidad metodologías y técnicas especiales (Antošová, 2013b) para incluir en el análisis las áreas rurales de frontera de la República Checa. Estos destinos fueron afectados por la Segunda Guerra Mundial y posteriormente durante el régimen totalitario. Se pretende encontrar enfoques teóricos que ayuden a responder la pregunta principal: ¿Cómo preservar y desarrollar las zonas de frontera más pobres de la República Checa?

\section{Descripción de la región de Liberec}

Los conceptos de las investigaciones sobre el desarrollo del turismo rural orientaron el trabajo práctico previo de este artículo, que incluyó visitas de campo y la aplicación de las encuestas a turistas, funcionarios y habitantes relacionados con la región checa de Liberec. La información recopilada pretende ilustrar la percepción de los turistas al visitar los atractivos relevantes de la región y descubrir el efecto de las ayudas europeas para la financiación de proyectos específicos de incidencia local.

Para efectos de este análisis, la región de Liberec es entendida como la demarcación político-administrativa definida en las cartografías oficiales checas; en ese sentido, nuestro enfoque deja intacto el territorio previamente definido en los respectivos mapas. Según la metodología OCDE (Organización para la Cooperación y el Desarrollo Económico, 20II), Liberec se caracteriza como una región mixta. Se encuentra ubicada en el norte de la República Checa, posee una 
frontera al norte que se extiende $20 \mathrm{~km}$ con Alemania y enlaza la frontera con Polonia en una longitud de $130 \mathrm{~km}$.

Esta región está formada por los siguientes distritos: Česká Lípa (con io ciudades y 46 municipios); Jablonec nad Nisou (con 3 ciudades y 30 municipios); Liberec (con 7 ciudades y 50 municipios); y Semily (6 ciudades y 6r municipios). La región de Liberec representa solo 4\% del territorio de la República Checa y a excepción de Praga, constituye la de menor tamaño del país $\left(3163 \mathrm{~km}^{2}\right)$. La tierra de cultivo ocupa $44.2 \%$ de su extensión, al tiempo que la cuota de tierra laborable (20.6 \%) está por debajo del promedio estatal. Es evidente además, que la cuota más alta de este territorio está dedicada a la actividad forestal (44.5\%), puesto que su geografía física es primordialmente montañosa, con características de cerros.

En la parte nordeste (en montañas Jizerské, Krkonoše y el somontano) el clima pertenece a una zona suave fría. La parte de la región en el oeste y el suroeste tiene condiciones de una zona suave cálida. Allí están localizados cinco zonas paisajísticas protegidas (el macizo central checo, las montañas Jizerské, las montañas Lužické, el paraíso checo, Kokořínsko), también incluye ocho reservas naturales nacionales, ocho monumentos naturales nacionales, 37 reservas naturales y 63 monumentos naturales.

Según la estadística checa el total de la población de esta región ascendió a 438527 habitantes a 30 de junio 2013 y según este índice demográfico se podía clasificar a Liberec como la segunda región menos poblada de la República Checa. Liberec tiene un carácter principalmente industrial y la actividad de procesamiento está especializada en la producción de vehículos y en los productos de goma y plástico. Una parte apreciable de la economía en esta región descansa en el turismo rural y turismo cultural, mientras que el sector agrícola funge solo como un sector suplementario. Posee una tradición histórica y culturalmente rica, que se refleja en la enorme cantidad de estructuras y patrimonios históricos y en algunas instalaciones culturales (galerías y museos regionales).

Se han desarrollado actividades e industrias atractivas, como el cristal y la bisutería que se ofrecen a visitantes en los museos de cristal en Nový Bor y Železný Brod, el museo de cristal y bisutería en Jablonec nad Nisou y Harrachov. Por otro lado se pueden mencionar atractivos adicionales como el museo de juguetes de madera en Jiřetín pod Bukovou, el museo de estatuas maduras en Františkov, los miradores en Tanvald y Kořenov, etc. Otros lugares de interés turístico son los destinos con caídas de agua como Harrachov, Josefův Dủl o paisajes culturales y 
naturales en Železný Brod, Jablonec nad Jizerou, Vysoké nad Jizerou, etc. Estas áreas son interesantes por su notoriedad en materia de turismo rural y cultural, su extraordinario paisaje, con su formación natural y vistas, los monumentos históricos y culturales en la región se convierten en metas para visitantes del extranjero y para residentes.

La mayoría de actividades están desarrolladas, principalmente, en función del turismo alternativo en sitios específicos (montañas Krkonoše, parte oeste, montañas Jizerské, Turnov, paraíso checo, Doksy y su externo, montañas Lužické y Podkrkonoší). Durante los dos primeros trimestres de 2013 fueron a esta región 337000 visitantes que dispusieron de 37000 posibilidades de camas en las diversas categorías de alojamiento. Los pasos fronterizos en carreteras y vías férreas, así como los pasos fronterizos peatonales en los campos limítrofes más pequeños, facilitan el desarrollo del turismo rural y cultural. En la región Liberec interviene también la eurorregión Neisse-Nisa-Nysa.

Liberec se constituyó en la primera eurorregión creada en la frontera checa y con la coincidencia de tres zonas de frontera de la República Checa, Alemania y Polonia. En la parte de la República Checa se encuentran ubicados izi municipios (para el año 20II). El desarrollo del turismo rural depende en su totalidad de los proyectos implementados con objetivos de larga sostenibilidad (por ejemplo, el mantenimiento de patrimonios y apoyos al turismo rural). La mayoría de estos proyectos en los destinos investigados de la región Liberec, están apoyados por fondos de la Unión Europea (Fondo Europeo de Desarrollo Regional [FEDER/ERDF, por sus siglas en inglés] con 60.7\%; Fondo de Cohesión [CF, por sus siglas en inglés] con 5.4\%; Fondo Social Europeo [ESF, por sus siglas en inglés] con 7.1\%; Fondo Europeo Agrícola de Desarrollo Rural [FEADER/EAFRD, por sus siglas en inglés] 26.8\%), en sus programas relevantes ${ }^{4}$ sobre este tema. Por su parte, el Ministerio de Desarrollo Local de la República Checa cada año organiza un concurso denominado Pueblo del Año, que concede premios a destinos exitosos en materia de turismos.

\footnotetext{
4 Incluye el Programa Integrado Operacional 4I.4\%, Programa Operacional [OP] objetivo polaco checo 10.9\%, OP transporte $0.7 \%$, OP recursos humanos y empleo $4.2 \%$, OP negocio e inovaciones $5.0 \%$, OP pesca $0.2 \%$, OP investigación y evolución en inovaciones $0.3 \%$, OP de educación y competitividad 24. $1 \%$ y OP de ambientales $13.3 \%$.

5 En lo regional, en las actividades de juventud, en cuidados de zonas verdes y medio ambiente, en cooperación entre municipios y empresa agrícola, en la vida social local y sociedad civil, en municipio y también premios a edificios como, por ejemplo, el Programa de Renovaciones en Municipios. Durante años 2007-2013 en la región Liberec fueron condecorados como destinos turísticos los siguientes municipios:
} 
Con respecto a la política turística, existe una organización institucional compuesta por la Secretaría Regional de Liberec y por un conjunto de secretarías municipales. Aunque en teoría debería existir una cooperación multinivel, en la práctica la Secretaría Regional de Liberec, al tener más jerarquía, ejecuta recursos más cuantiosos y tiene más peso ante las instancias nacionales.

Por las razones expuestas, en las siguientes partes de la investigación se pretende interpretar y describir los resultados de la información procedente de las encuesta con base en el análisis de los casos de "proyectos exitosos". Los destinos investigados fueron elegidos según su notoriedad en el turismo rural, sus impactos positivos y según la larga sostenibilidad de los proyectos implementados. La promoción del turismo es una alternativa para el desarrollo de la región de Liberec, una zona cuyos indicadores económicos y sociales la ubican en posiciones inferiores a las del promedio de la República Checa. Sus habitantes viven predominantemente en las zonas urbanas, de modo tal que la población urbana representa alrededor de $78 \%$ del total.

Por otro lado, la evolución de los indicadores en la primera década del presente siglo refleja un claro deterioro de las condiciones de la población de la región. En efecto, en $20 \mathrm{Io} \mathrm{el} \mathrm{PIB} \mathrm{per} \mathrm{cápita} \mathrm{ascendió} \mathrm{(Cuadro} \mathrm{I)} \mathrm{a} 268480$ coronas checas, lo que representó 75\% del promedio de todo el país. Según este indicador el ingreso promedio de los habitantes de Liberec se encuentra $25 \%$ por debajo del resto de regiones y lo más preocupante es que a lo largo de la última década, año tras año la riqueza per cápita generada en la región es, en términos relativos, cada vez más reducida con respecto al total del país. Asimismo, las cosechas agrícolas registraron especial abundancia a mediados de la primera década del siglo, pero con una reducción en los años finales. En el caso de los cereales en 2004, la cosecha alcanzó un pico de 132.3 miles de toneladas con posteriores descensos y nuevos incrementos, para acabar la década con una cosecha de 93.3 miles de toneladas en 2010. La cosecha de papas tuvo un descenso más continuado al pasar en 2002 de 13000 a 5000 toneladas en 2010.

Con respecto al mercado laboral, la tasa de desempleo ha registrado una tendencia en alza durante los años de aplicación de ambas metodologías. En 2010 se elevó a 10.5\% de la Población Activa, lo que indica un incremento de la desocupación en la región con respecto a lo ocurrido en los primeros años de la década y al año 2009, cuando hubo una tasa de desempleo particularmente alta.

Zahrádky, Pertoltice, Nová Ves nad Nisou, Pulečný, Rádlo, Polevsko. 


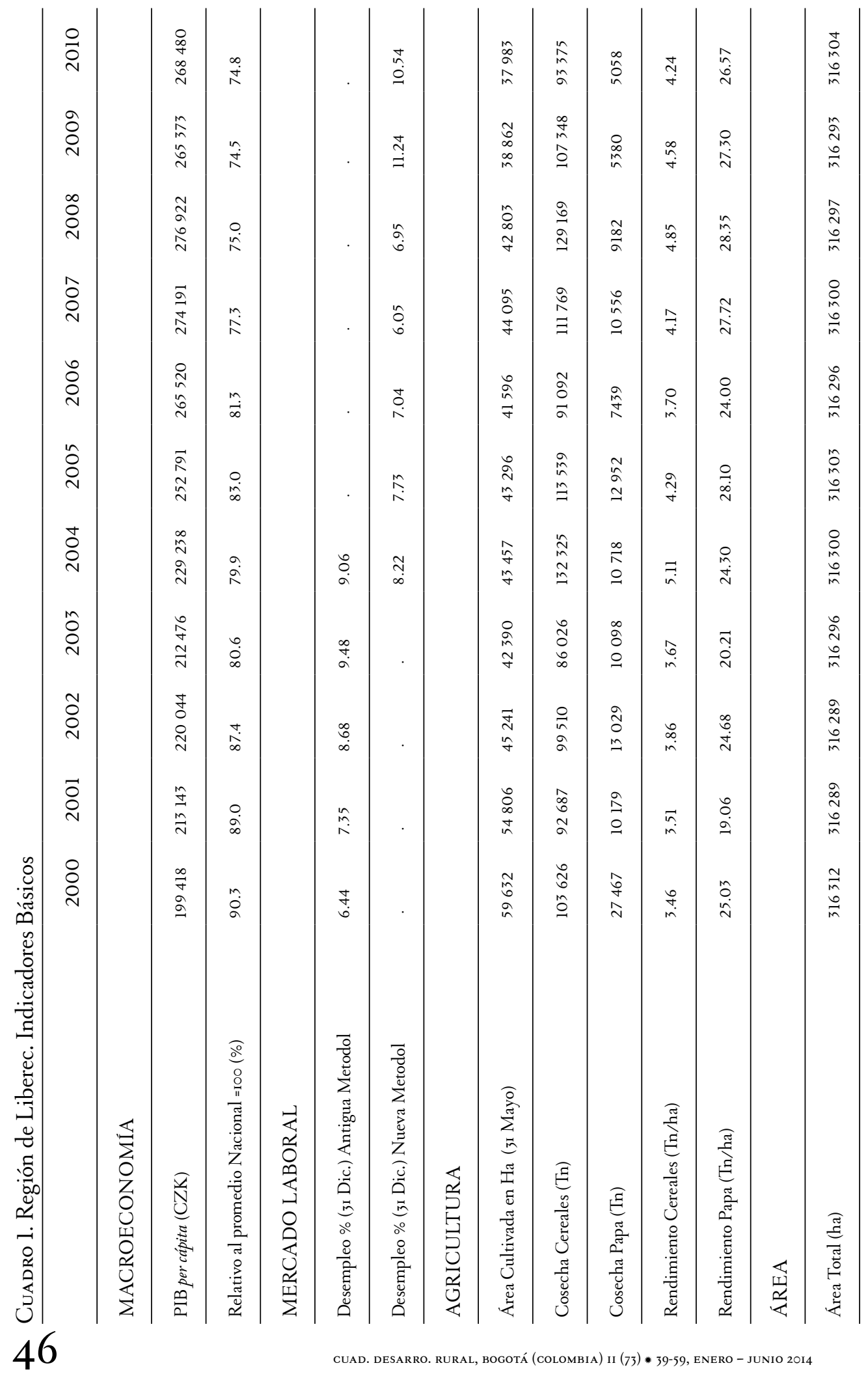




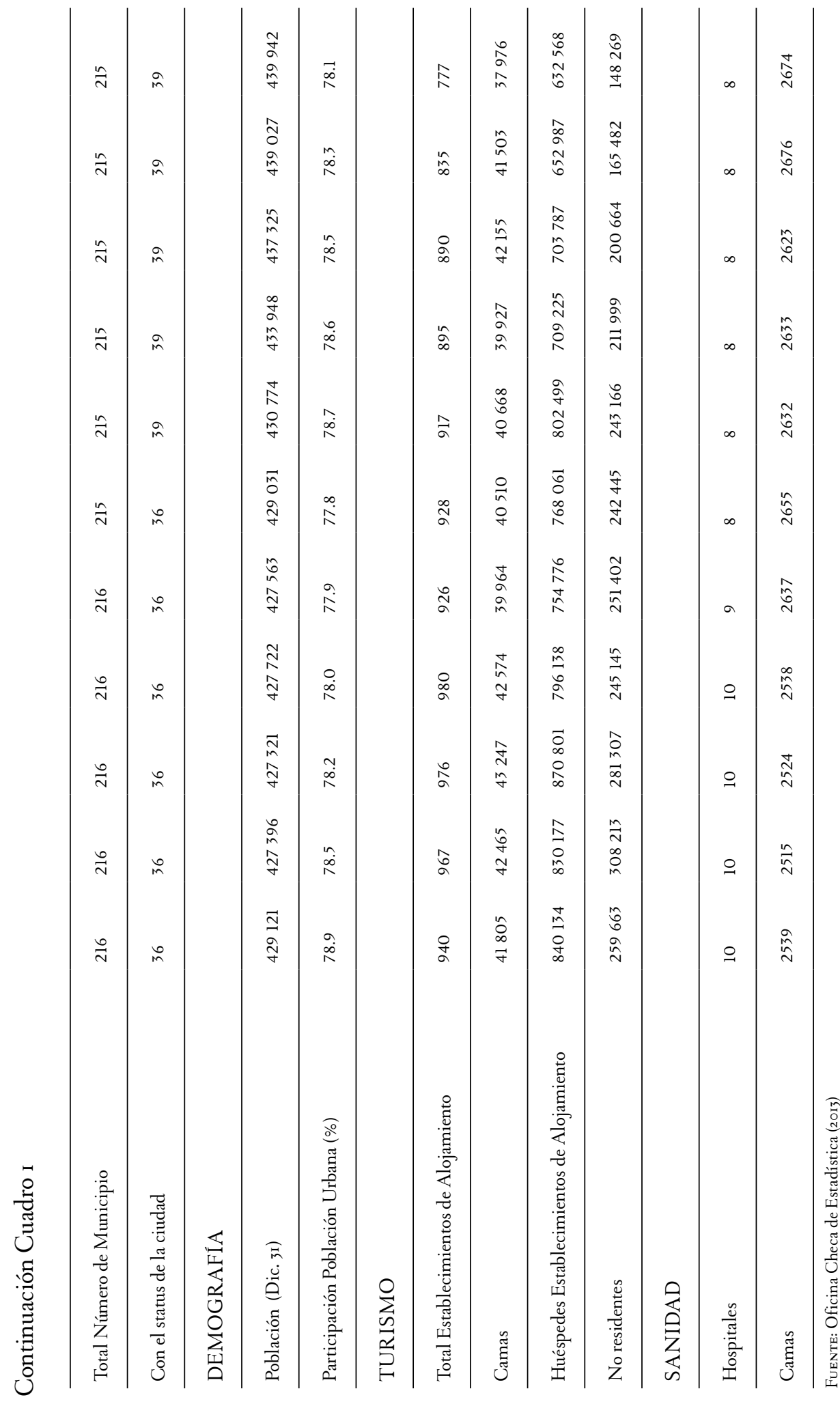




\section{Metodología}

En el estudio sobre el turismo cultural rural en la región Liberec se aplica un enfoque multidisciplinario, basado en las metodologías de multiplicadores y de encuestas a los visitantes, alcaldes y habitantes. Los métodos múltiples son usados para examinar diferentes perspectivas sobre el éxito entre proyectos investigados en destinos elegidos sobre el mismo tema de la investigación (Finn, Elliot-White y Walton, 2000) en turismo rural, mientras que el uso de métodos combinados aplicados a los turistas, alcaldes y habitantes, implicó el uso simultáneo de diferentes métodos durante el proceso de investigación. Con respecto al tema que nos ocupa, los datos básicos fueron recolectados desde octubre 201 ir hasta octubre 2013 en dos etapas.

La primera etapa incluyó la investigación secundaria y la segunda desarrolló la organización de la investigación primaria. Los datos completados y tratados en ambas fases son usados para formular recomendaciones y diseños en el turismo cultural rural (discusiones intensas con autogestores locales de los lugares, directores de destinos turísticos organizadas con Ministerio de Agrícola para la región de Liberec). La segunda etapa fue presentada con la captura de datos primarios por parte del investigador, quien observó durante octubre 20II-octubre 2013 los proyectos implementados en Liberec. Durante el trabajo de campo se indagó acerca de los impactos positivos y negativos de proyectos implementados en destinos elegidos según respuestas de habitantes, alcaldes y turistas. Esta observación se efectuó con seis técnicas investigadas (uso de la triangulación):

* El estudio de documentos se especializó en dos tipos de datos: fuentes de las estadísticas checas y otros documentos facilitados, datos sobre los municipios y sus alrededores (dónde se implementaron los proyectos y los planes para la implementación de futuros desarrollos); documentos sobre la realización de proyectos, en especial, notas de prensa publicadas y anotaciones del equipo de realizaciones.

* Las observaciones de los no participantes se recogieron en tres encuentros con 22 alcaldes y un organizador del Ministerio de Agricultura (Departamento de la Región Liberec), con ocasión del aniversario de los premios otorgados a los destinos exitosos.

* Cuestionarios estandarizados con respuestas voluntarias fueron enviados vía correo electrónico a los 216 alcaldes. Los mandatarios tuvieron cuatro opciones para diligenciar este cuestionario: en la página web especialmente preparada para esta investigación; vía correo electrónico diligenciado de manera directa sobre el 
cuerpo del mensaje; cuestionario escaneado y enviado como adjunto vía correo electrónico; o responder las preguntas por teléfono.

* Cuestionarios estandarizados con habitantes (en total I62) en los destinos elegidos de región Liberec, según la densidad de población en cada destino (mínimo 6-máximo I5).

* Encuestas estandarizadas a turistas durante dos temporadas invierno/verano 2012-2013. El total de turistas ${ }^{6}$ le respondió a un investigador especializado en turismo rural y desarrollo de destinos en áreas rurales. Los participantes pudieron expresar sus motivos y expectativas en cada destino observado ( 23 lugares en total, considerados atractivos ${ }^{7}$ y menos ${ }^{8}$ atractivos).

* Entrevistas cualitativas con algunos iniciadores de proyectos implementados en destinos exitosos: un empresario autónomo, tres directores de equipos acciones locales, cinco representantes de autogestores del lugares.

Los datos de las encuestas estandarizadas y semiestandarizadas fueron procesados con el programa SPSS. Los datos primarios de las entrevistas cualitativas fueron evaluados por los investigadores con un enfoque tanto objetivo como imparcial, que permitió determinar las variables ${ }^{9}$. El tratamiento con estas variables fue tanto objetivado como subjetivado, sobre todo en cuanto a los impactos de la identidad local. Sin embargo, se observó que no era posible aplicar el procedimiento exacto usado normalmente. En la última etapa de este artículo se hace una discusión y evaluación de buenas y malas prácticas realizadas según este enfoque. Debe decirse que el cuestionario no incluyó preguntas acerca del estrato socioeconómico ni la condición económica del turista porque se consideró que no eran relevantes para el propósito de la investigación.

\footnotetext{
6 I89 participantes en el turismo rural que estuviesen fuera de su residencia más de una noche (84\%) o menos de una noche ( $16 \%)$.

7 Bozkovské dolomity, Dvoračky, Harrachov, Jilemnice, Jiřetín pod Bukovou,Josefův Důl, Malá Skála, Rezek, Rokytnice nad Jizerou, Tanvaldský špičák, Železný Brod.

8 Desná, Františkov, Horní Branná, Jablonec nad Jizerou, Kořenov, Martinice v Krkonoších, Návarov, Roudnice, Smržovka, Tanvald, Trojmezí, Vysoké nad Jizerou.

9 Solicitantes y sus compañeros de las actividades (cooperación); los problemas durante el diseño de proyecto; buenas y malas experiencias durante la realización de proyecto; los resultados, expectativas y sus impactos en el proyecto (con objetivo de la revalorización de patrimonio cultural y su desarrollo en el turismo cultural rural); las recomendaciones eventuales (para los otros iniciadores o por otro trabajo).
} 


\section{Resultados de análisis}

La información recopilada para la investigación permite hacer un análisis desde diversos puntos de vista y enfoques. En primer lugar, se tienen datos de los fondos asignados desde Bruselas para el desarrollo local, por medio del financiamiento directo de algunos proyectos específicos. Asimismo, como ya se explicó, se recopiló información de los agentes locales sobre su implicación en el desarrollo de los proyectos con financiación europea, mediante encuestas que indagaban sobre la opinión de alcaldes locales y habitantes de los sitios. También se consultó la opinión de los turistas.

La síntesis de los tres puntos de vista permite retroalimentar la opinión de los turistas, habitantes y los alcaldes en cuanto a la necesidad de emprender nuevos proyectos. En efecto, a partir de las recomendaciones de los turistas se pueden identificar necesidades y aplicar mejoras para el desarrollo del turismo cultural rural, que pueden ser acogidas por las administraciones públicas y constituirse en potenciales proyectos susceptibles de financiación por parte de la Unión Europea (UE).

A tenor de la situación general, la mejora de los atractivos turísticos y su incorporación al mercado por medio de una explotación sustentable, es una vía para mejorar las condiciones de vida de los habitantes de la zona y para diversificar la economía regional. Las mismas estadísticas reportan que los alojamientos de la zona se han reducido de forma paulatina durante la primera década del presente siglo: de un total de 980 en 2003 , el número ha caído a solo 777 establecimientos en 2010 . Este fenómeno, como es lógico, implica la reducción del número de camas disponibles, las cuales pasaron de alrededor de 43000 en los tres primeros años del siglo XXI, a solo 37900 en el año 20ro. De igual modo, se ha notado un descenso del número de huéspedes, que pasaron de 871 000 en 2002 a solo 632000 en 2010.

Los indicadores descritos dan cuenta de un deterioro de la situación de la región, a pesar de que durante el mismo período se han recibido ayudas provenientes de la UE. Esto lleva a pensar que los fondos estructurales europeos deben entenderse como un mero complemento al proceso de desarrollo regional. Por tanto, el crecimiento económico de Liberec debe estar basado en el potencial productivo regional, la innovación, sus propios recursos y su talento humano.

Cuadro 2. Región de Liberec. Fondos provenientes de la Unión Europea (en millones de euros corrientes)

\begin{tabular}{ccccc}
\hline 2008 & 2009 & 2010 & 2011 & 2012 \\
\hline 60.1 & 268.5 & 90.9 & 104.8 & 5.8 \\
\hline
\end{tabular}

FuENTE: elaboración propia según encuestas aplicadas a alcaldes, quienes consultaron sus bases de datos municipales 
En este contexto debe decirse que la Unión Europea ha destinado fondos al desarrollo de la región de Liberec, que han sido ejecutados en diferentes tipos de proyectos, vinculados con educación, innovación, cultura, planificación del desarrollo, energía, desarrollo productivo, entre otros. La información que se tiene muestra que los montos provenientes de Bruselas ascendieron (Cuadro 2) en 2008 a 60.r millones de euros y en 2012 se redujeron a 5.8 millones de euros. Como se ha dicho, estos flujos podrían causar un efecto sobre el crecimiento de forma indirecta, mediante la potencialización de los factores fundamentales del crecimiento en la región de Liberec.

\section{I. Encuestas con habitantes}

A continuación se presentan los resultados porcentuales de cada una de las preguntas que fueron formuladas a los habitantes sobre los proyectos ${ }^{10}$ implementados (aleatorio según posibilidades en cada destino). Rokytnice nad Jizerou (I4), Hodkovice nad Mohelkou (I5), Jablonné v Podještědí (I4), Osečná (II), Křižany (I4), Tatobity (I0), Karlovice (7), Kacanovy (I0), Frýdlant (I4), Liberec (Iz), Dětřichov (6), Heřmanice (6), Višňová (13), Semily (4) y Nová Ves nad Nisou (IO). Las respuestas incluyen siete tipos de proyectos elegidos en cada destino, que fueron llevados a cabo durante entre 2007 y 2012.

Cuadro 3. Encuesta de habitantes

Características de PARTICIPACión EN El PROYECTO

I. Participación en el proyecto: Absolutamente no (80.1\%); En menor medida (8.1\%); En mayor medida (4.3\%); De manera importante (7.5\%)

II. Deseo de participar en el proyecto: Absolutamente no (40.4\%); En menor medida (29.8\%); En mayor medida (12.4\%); De manera importante (16.1\%); No lo sé (0.6\%); Datos impropios (0.6\%)

III. Razones para inbibirse del proyecto: No había una razón (9.9\%); Había razones, pero no era la oportunidad (8.7\%); Totalmente, no sé qué se puede contratar (2.5\%); Tengo otros intereses, yo no me intereso por los acontecimientos en municipio (17.4\%); No lo sé $(2.5 \%)$; No es relevante $(60 \%)$

IV. Reconocimiento del proyecto antes de la implementación Absolutamente no (I4.9\%); En menor medida (28.6\%); En mayor medida (18.0\%); De manera importante (20.5\%); No lo sé (18.0\%)

Fuente: Resultados de la investigación según los cuestionarios aplicados a los habitantes

Io En cada tipo de proyecto, el investigador codificó estos temas: I) Renovación de edificios (colegio, guardería, museo, librería, galería) (26); 2) Ruta de bicicletas y otras actividades para turismo (33); 3) Edificio cultural/centro social (38); 4) Renovación/reconstrucción de la infraestructura (7); 5 ) Parque infantil/pistas, plazas para el tiempo libre (17); 6) Parque/las áreas públicas/vegetación/alamedas (22); y 7) Bases para actividades educativas (museos, librerías, cine, galerías) (I8). 
En las encuestas aplicadas a los habitantes de la región se indagó sobre el grado de conocimiento e interés que les despertaron los proyectos. La revisión de las respuestas (Cuadro 3) revela que la implicación de la población en estos fue verdaderamente escasa. Entre los encuestados, $80 \%$ respondió que no participaron en el proyecto desarrollado en su municipio, mientras que alrededor de $70 \%$ expresó que no tenía deseos de participar o que su deseo era muy débil. Por otra parte, más de $27 \%$ de los habitantes no planteaba una postura acerca del proyecto y declaraba no saber acerca del tema.

\subsection{Encuestas a alcaldes}

Durante la investigación se aplicó una encuesta a los alcaldes de i8 municipios, en la que se indagaba acerca de la importancia de la financiación europea en proyectos de impacto local. En los municipios sobre los que existe información se adelantaron proyectos por un valor aproximado de 6.5 millones de euros, los cuales contaron con una financiación proveniente de fondos europeos por un monto de 4.9 millones de euros (Gráfico I).

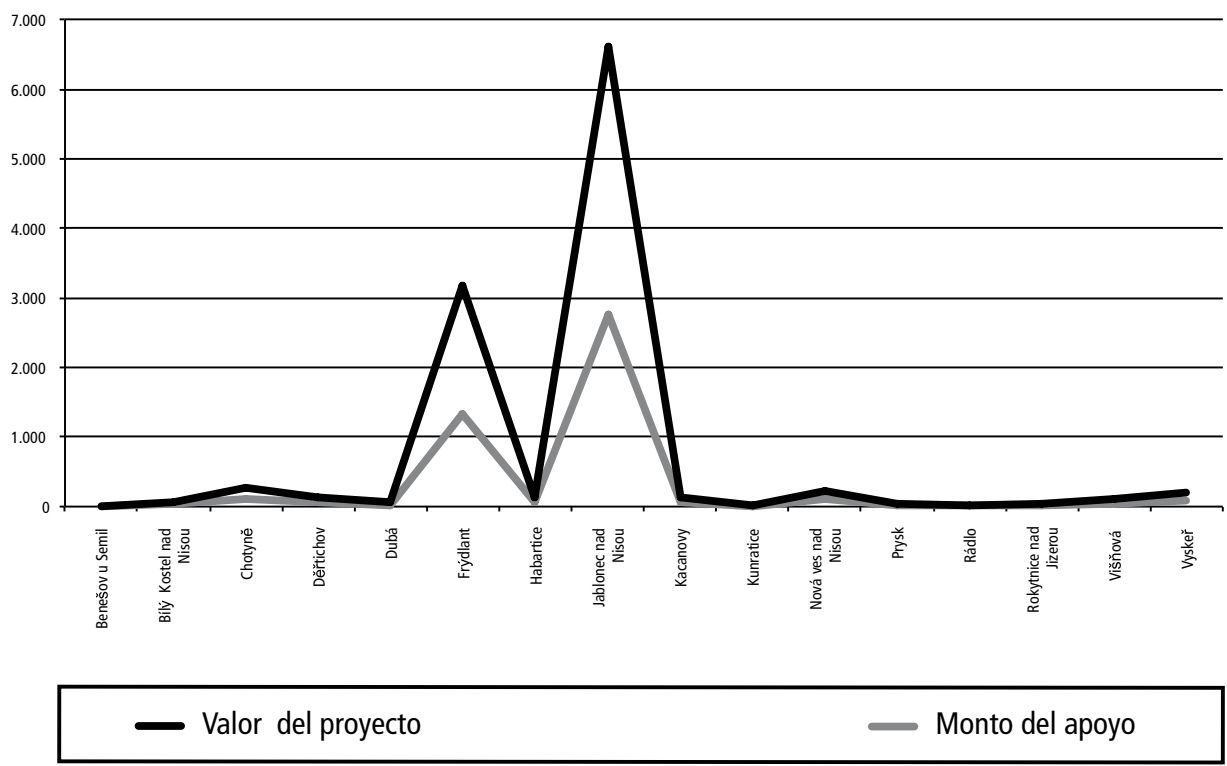

GrÁfico I. Municipios de la región de Liberec. Proyectos municipales y financiación de la Unión Europea

FuENTE: Encuesta de alcaldes, quienes consultaron sus bases de datos municipales 
Datos generales de MUNicipio

I. Cantidad de los proyectos apoyados por fondos UE entre 2007-2012: o proyectos (I6.7\%); I-5 proyectos (6I.2\%); 6-8 proyectos (II.2\%); I4-28 proyectos (II. $2 \%$ )

II. Beneficiarios de los apoyos anteriores entre 2000/2004-2006: $\quad$ Sí (44.4\%); No (55.6\%)

III. Beneficiarios de otros fondos de la UE entre 2007-2013: $\quad$ Sí (66.7\%); No (33.3\%)

IV. Solicitudes de apoyo de fondos UE rechazadas: $\quad$ Sí (55.6\%); No (44.4\%)

FuENTE: Resultados de la investigación según los cuestionarios de los alcaldes, quiénes consultaron en sus bases de datos municipales

Los municipios más favorecidos con los recursos europeos fueron Jablonec nad Nisou y Frýdlant cuyos proyectos se concentraron en programas vinculados al medioambiente, desarrollo regional y construcción de instalaciones físicas. El resto ejecutó proyectos financiados por la Unión Europea con valores inferiores (Cuadro 4), que abarcaron la construcción de infraestructura, compra de automotores, programas para jubilados, etc.

Dado que hubo una escasa colaboración de los alcaldes en cuanto a participar en la investigación, se registró un bajo nivel de respuesta de este tipo de encuestas. Con respecto a los métodos y técnicas usadas en esta medición, es necesario decir que respondieron solo i8 alcaldes, de un total 216, mediante una entrevista convenida con antelación por vía telefónica. Por tal razón se resolvió integrar un observador independiente que participó en octubre de 2013 en tres excursiones" Durante las discusiones y presentaciones formales e informales entre los alcaldes y representantes de municipios en esta región, participantes en las excursiones, se aludió a las causas que explican la ausencia de algunos municipios en el proceso de diligenciamiento de las encuestas.

Entre las razones expuestas se encuentran: el bajo perfil educativo de los mandatarios locales, que no corresponde a la altura de sus posiciones (la mayoría de alcaldes solo tiene enseñanza secundaria); el desinterés de los funcionarios en participar; la falta de tiempo (para algunos alcaldes es obligatorio alternar sus funciones en municipio con el ejercicio de otra profesión); la ocurrencia de inundaciones frecuentes en municipios; la desconfianza ante los agentes externos al municipio; la experiencia sobre complicaciones anteriores a la hora de demostrar los apoyos de la UE; imprudentes enfoques de alcaldes en la gestión de los recursos ${ }^{12} ; \mathrm{y}$, la poca importancia concedida al diligenciamiento de las encuestas.

II Se trató de dos excursiones organizadas por el Ministerio de Agricultura, concretamente el Departamento de la región Liberec, para los representantes de los municipios. En estas se desarrollaron temas de desarrollo rural y los proyectos implementados en los destinos elegidos.

I2 El 22 de octubre de 2013, el periódico MF Dnes publicó una nota sobre la acusación contra 23 representantes municipales de Liberec por un delito penal, debido a la venta no autorizada de terrenos. Por esta razón, el 
Cuadro 5. Impactos negativos y positivos según encuestas de habitantes y alcaldes

EN SU OPINIÓN, LA REALIZACIÓN DE ESTE PROYECTO EN SU MUNICIPIO:

\begin{tabular}{|c|c|c|c|c|c|c|}
\hline \multicolumn{2}{|c|}{$\begin{array}{l}\text { IMPACTOS POSITIVOS/NEGATIVOS } \\
\text { DE LA REALIZACIÓN }\end{array}$} & \multirow{2}{*}{$\begin{array}{c}\text { Absolutamente } \\
\text { No } \\
20.5 \%\end{array}$} & \multirow{2}{*}{$\begin{array}{c}\text { EN } \\
\text { MENOR } \\
\text { MEDIDA } \\
30.4 \%\end{array}$} & \multirow{2}{*}{$\begin{array}{c}\text { EN MAYOR } \\
\text { MEDIDA } \\
27.3 \%\end{array}$} & \multirow{2}{*}{$\begin{array}{l}\text { DE MANERA } \\
\text { IMPORTANTE } \\
2 \text { I.I\% }\end{array}$} & \multirow{2}{*}{$\begin{array}{c}\text { No } \\
\text { LO SÉ } \\
0.6 \%\end{array}$} \\
\hline I. Aumentó & Habitantes & & & & & \\
\hline visitantes & Alcaldes & $38.9 \%$ & $38.9 \%$ & $5.6 \%$ & $5.6 \%$ & II.I \% \\
\hline \multirow{2}{*}{$\begin{array}{l}\text { II. Posibilitó una } \\
\text { actividad nueva }\end{array}$} & Habitantes & $26.1 \%$ & $23.0 \%$ & $23.0 \%$ & $28.0 \%$ & $0.0 \%$ \\
\hline & Alcaldes & $16.7 \%$ & II.I \% & $27.8 \%$ & $33.3 \%$ & II.I \% \\
\hline \multirow{2}{*}{$\begin{array}{l}\text { III. Mejoró la } \\
\text { infraestructura }\end{array}$} & Habitantes & $77.0 \%$ & $10.6 \%$ & $7.5 \%$ & $5.0 \%$ & $0.0 \%$ \\
\hline & Alcaldes & $66.7 \%$ & $5.6 \%$ & $16.7 \%$ & $0.0 \%$ & II.I \% \\
\hline \multirow{2}{*}{$\begin{array}{l}\text { IV. Mejoró el } \\
\text { diseño de nuestro } \\
\text { municipio }\end{array}$} & Habitantes & $20.5 \%$ & $24.8 \%$ & $21.7 \%$ & $32.3 \%$ & $0.6 \%$ \\
\hline & Alcaldes & $55.6 \%$ & $0.0 \%$ & $0.0 \%$ & $33.3 \%$ & II.I \% \\
\hline \multirow{2}{*}{$\begin{array}{l}\text { V. Contribuyó } \\
\text { el aumento del } \\
\text { nivel de empleo }\end{array}$} & Habitantes & $73.9 \%$ & $\mathrm{I} 6.8 \%$ & $5.6 \%$ & $2.5 \%$ & $1.2 \%$ \\
\hline & Alcaldes & $72.2 \%$ & $16.7 \%$ & $0.0 \%$ & $0.0 \%$ & II.I \% \\
\hline
\end{tabular}

FuENTE: Resultados de la investigación según cuestionarios aplicados a alcaldes y habitantes

\subsection{Resultados e impactos de proyectos según alcaldes y habitantes}

De otro lado, la investigación recopiló datos sobre el impacto de los proyectos desarrollados en el medio local, razón por la cual se indagó entre los alcaldes y habitantes acerca de cómo evalúan los efectos de la realización de los proyectos en el medio local. De las respuestas se deduce que la evaluación de los funcionarios con respecto al impacto de los proyectos es más positiva (Cuadro 5), comparada con las respuestas dadas por los habitantes.

\subsection{Encuestas de turistas}

Uno de los propósitos de la investigación fue evaluar la capacidad para desarrollar actividades turísticas y dar origen a dinámicas regionales que promovieran la actividad económica y el empleo. Asimismo, era de gran interés conocer la percepción de los turistas con respecto a las infraestructuras y la organización turística de los atractivos de la zona. Por lo anterior, dentro de la metodología de estudio se aplicó una encuesta que contenía preguntas relativas a la experiencia del visitante en su recorrido por los diferentes puntos de la geografía de Liberec.

año pasado el municipio perdió más de 135000 euros. Este caso es un ejemplo de los equivocados manejos de algunos representantes de municipios en esta región, en particular, en la ciudad de Liberec. 


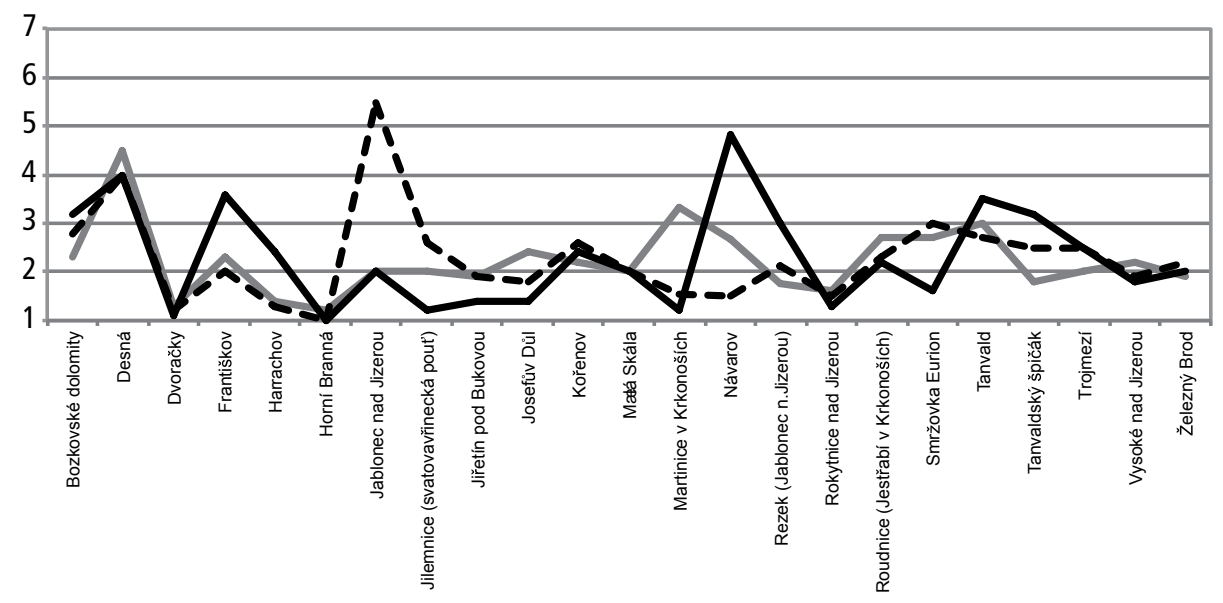

— Experiencia memorable - - Cumplió mis expectativas — Le gustaría visitarlo nuevamente

Gráfico 2. Experiencia memorable en los destinos elegidos

FuENTE: Encuestas directas a turistas

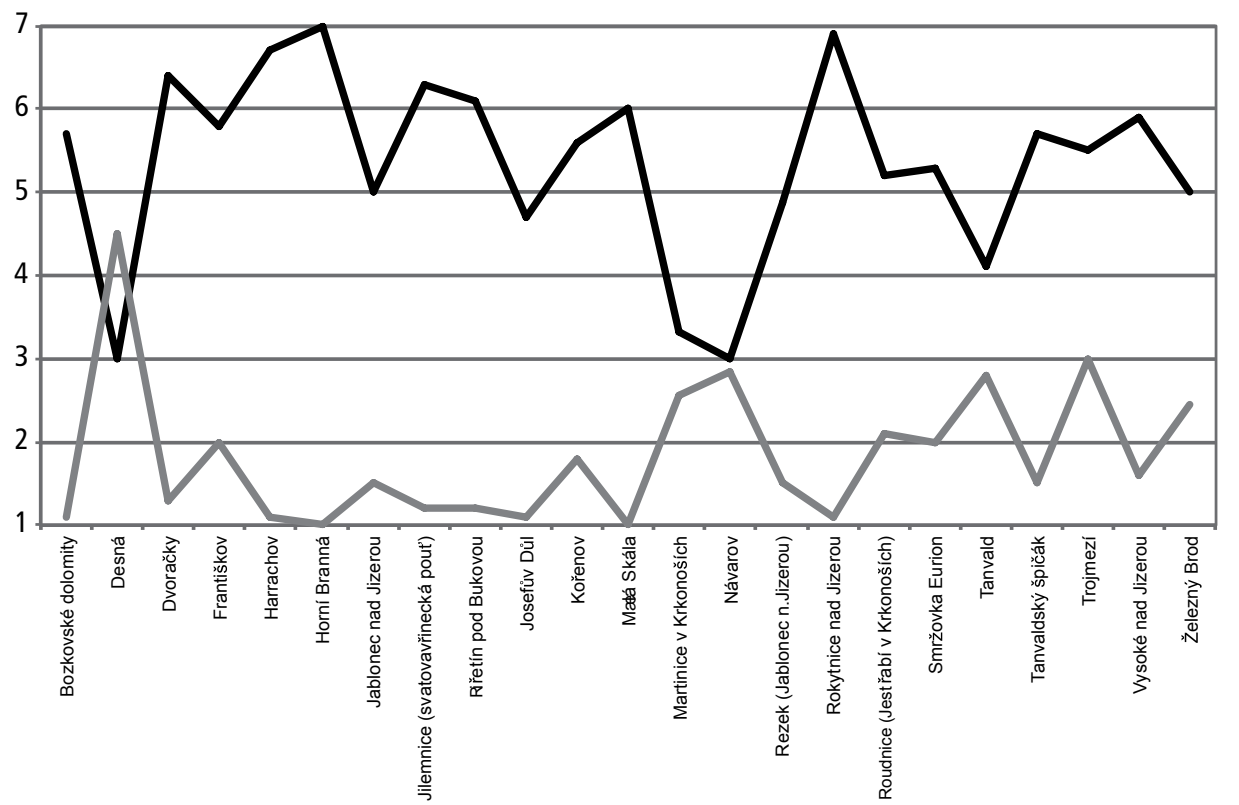

No tuvo efecto

Lo recomendaría a otros

Gráfico 3. Las expectativas de los turistas

Fuente: Encuestas directas a turistas 
Algunas preguntas estaban enfocadas a captar la percepción del turista sobre su experiencia en la zona y en estas se pedía calificar, según una escala, la impresión que la persona obtuvo en su visita. Ante una afirmación determinada, los turistas expresaban su acuerdo o desacuerdo con base en una puntuación determinada. Los diferentes grados de calificación abarcaban valores discretos entre i y 7, donde I correspondía a una situación en que los turistas estaban muy de acuerdo con la afirmación planteada, y 7 en que la persona se manifestaba en total desacuerdo con la afirmación.

Los gráficos 2 y 3 representan los resultados sobre el acuerdo o desacuerdo con respecto a algunas experiencias de la visita. En tres preguntas se indagaba si la visita a los atractivos había sido una experiencia memorable, si había cumplido las expectativas y si permanecía el deseo de repetir la visita. Al observar los gráficos, los turistas sintieron una fuerte afinidad con las afirmaciones propuestas en la mayoría de destinos. En el caso de Jablonec nad Jizerou, pocos se inclinaron por la afirmación sobre experiencia memorable (Gráfico 2). En Tanvald se registró el acuerdo en menor medida con los aspectos llamativos de ese destino. Las opiniones de insatisfacción pudieron deberse a que en el primer destino, Jablonec nad Jizerou, no se había restaurado un hotel ubicado en la zona céntrica para el servicio de los turistas. En Tanvald una vía vehicular de comunicación entre la República Checa y Polonia causa contaminación urbana y congestión en la circunvalación del municipio.

Otras dos preguntas fueron diligenciadas por los turistas. En una de ellas se indagó sobre su intención de regresar al sitio visitado, y la otra se formuló con una redacción inversa, en términos de si el lugar visitado no causó efecto alguno. Con respecto a los destinos, dos de ellos Horní Branná y Rokytnice nad Jizerou (Gráfico 3) causaron un importante efecto en los turistas, y al mismo tiempo se reveló que los turistas lo recomendarían a otros para visitarlos.

\section{Conclusiones}

El turismo puede ser explotado como una fuente importante de empleo y desarrollo productivo en la región de Liberec, con una adecuada gestión y planificación. Esta actividad surge como una alternativa importante para desarrollar las áreas menos dinámicas, con la aplicación de políticas apropiadas, el suficiente desarrollo de la infraestructura turística, la movilización de capitales y la calificación de la mano de obra local. Los fondos de la Unión Europea no promueven en sí mismos el crecimiento de las regiones, sino que sirven de complemento a los factores fundamentales asociados de las fuerzas económicas locales. La financiación 
europea debe generar sinergias con los factores locales como el capital humano, la innovación, la acumulación de capital físico y la tecnología, para así tener un efecto importante en el crecimiento.

En este contexto la política de la UE pretende tener un enfoque que privilegia los ámbitos local y regional. Sin embargo, la financiación comunitaria ha demostrado tener múltiples problemas. En primer lugar, existen muchas dificultades entre las autoridades locales para generar recursos propios con el fin de prefinanciar los proyectos, además de los interminables trámites burocráticos. Adicionalmente, la persistencia de la crisis económica en Europa ha significado importantes recortes en la asignación de recursos destinados al desarrollo local. A la hora de implementar proyectos de desarrollo, sin importar de dónde provengan los fondos, se requiere una participación más directa de la población, y que ésta perciba el beneficio de las acciones emprendidas. Según las encuestas cualitativas con agentes iniciadores ${ }^{13}$, se observó que las buenas experiencias contaron con la cooperación entre los iniciadores, los equipos de acción local y las administraciones de los proyectos.

Un caso de estrecha cooperación entre iniciadores y las administraciones se presentó en Noruega ${ }^{14}$. Los representantes de las agencias de autogestión de los lugares y los empresarios estuvieron totalmente de acuerdo en la identificación de los problemas relacionados con la administración de los proyectos, con las inversiones financiadas por la UE y a veces con la cofinanciación de proyectos desarrollados en los destinos.

Según las experiencias de agentes iniciadores exitosos, la buena cooperación entre representantes de destinos rurales y los habitantes promueve el turismo rural y el desarrollo regional. Esta cooperación tiene su base principal en la fluida comunicación y la coincidencia de objetivos que posibilitan un trabajo común y propician el desarrollo de los destinos rurales y del turismo rural cultural. De acuerdo con las encuestas, los iniciadores recomendaron implementar estrategias turísticas en otros destinos no tan exitosos para su desarrollo, e identificaron como puntos importantes el mantenimiento del patrimonio o el desarrollo del turismo rural, la insistencia en la vocación turística y la definición de una visión de largo plazo con apoyo de la Unión Europea.

13 Eso incluye un empresario autónomo en Františkov, tres directores de equipos acciones locales-LAG Frýdlantsko, LAG Podralsko, LAG Turnovsko, y cinco representantes de autogestiones del lugares-Nová Ves nad Nisou, Pulečný, Rádlo, Vyskeř y Polevsko.

I4 Se aplica una ayuda estatal con las administraciones de proyectos, que funciona desde la etapa de planificación hasta su implementación, de forma gratuita para los destinos. 
Además, se recomendó la inscripción de proyectos locales en concursos, como el organizado por el Ministerio de Desarrollo Local de la República Checa, denominado Pueblo del Año, que da visibilidad a los resultados de los casos exitosos de comercialización de destinos rurales. Las encuestas de turistas permiten deducir que la región de Liberec posee un gran potencial para su progreso, si aprovecha su patrimonio cultural y natural para desarrollar algún tipo de turismo rural cultural. Para potenciar sus paisajes deberían combinarse los deseos y motivos de los representantes de autogestión de los lugares, con la participación de los habitantes de esos destinos, con el fin de promover sus identidades locales y productos turísticos.

Debe considerarse también la oportunidad de complementar los flujos de inversión con la búsqueda de apoyos provenientes de la Unión Europea, que serían remunerados con un flujo de ingresos futuros y que retribuirán en lo social a la comunidad en forma de desarrollo local. Las encuestas revelan una escasa implicación de los agentes locales en los proyectos. Este aspecto es muy importante para garantizar la sostenibilidad del desarrollo del destino turístico. Por tanto los proyectos turísticos deben incorporar la sostenibilidad ambiental, la conservación de patrimonio, la absorción de mano de obra local y la generación de encadenamientos económicos con el medio local, de manera que los agentes del entorno interpreten como provechosa la puesta en marcha de los proyectos turísticos.

\section{Referencias}

Antošová, G. (2013a). A Review of Quantitative and Qualitative Approaches and Usage of Researchers Terminology in Cultural Rural Development. Documento presentado en $4^{\text {th }}$ International PhD Students' Conference New Economic Challenges. Masaryk University, Brno.

Antošová, G. (20I3b). Multidisciplinary Determinants of Cultural Rural Development. The Keynote Usage of Determinants and Methods in Multidisciplinary Context. Documento presentado en $2^{\text {nd }}$ Electronic International Interdisciplinary Conference 2013. University of Žilina, Eslovaquia. Recuperado el 15 de Marzo 2013, de http://www.eiic.cz/EIIC-2013_ Proceedings.zip

Diario Mladá Fronta DNES, La región de Liberec. (22 de octubre 20I3).

Feiner, J. P., Mi, S. y Schmid, W. A. (2002). Sustainable Rural Development Based on Cultural Heritage: the Case of the Shaxi Valley Rehabilitation Project. DISP, I5I(4), 79-86. 
Finn, M., Elliot-White, M. y Walton, M. (2000). Tourism and Leisure Research Methods: Data Collection, Analysis and Interpretation. Londres: Pearson Education.

Garrod, B., Wornell, R. y Youell, R. (2006). Re-Conceptualising Rural Resources as Countryside Capital: the Case of Rural Tourism. Journal of Rural Studies, 22(I), II7-I28.

Gunn, C. (2002). Tourism Planning (4th edition). Nueva York: Taylor \& Francis.

Kausar, D. R. y Nishikawa, Y. (2010). Heritage Tourism in Rural Areas: Challenges for Improving Socioeconomic Impacts. Asia Pacific Journal of Tourism Research, $15(2)$, I95-213.

Kürker, Ö. y Dinçyürek, Ö. (2007). Sustainable Tourism as an Alternative to Mass Tourism Developments of Bafra, North Cyprus. Open House International, $32(4)$, I07-II8.

MacDonald, R. y Jolliffe, L. (2003). Cultural Rural Tourism: Evidence from Canada. Annals of Tourism Research, 30(2), 307-322.

Mason, P. (2003). Tourism, Planning and Management. Oxford: Butterworth-Heinemann Publications.

Ministerio de Desarrollo Local de la República Checa (2013). Pueblo del año. Recuperado el 23 de marzo 2013, de http://www.vesniceroku.cz/uvod

Niezgoda, A. y Zmyślony, P. (2003). Identifying Determinants of Development of Rural Tourist Destinations in Poland. Tourism, 5 I(4), 443-454.

Oficina Checa de Estadística (2013). Administración de la región Liberec. Recuperado el 23 de marzo 2013, de http://notes2.czso.cz/xl/redakce.nsf/i/home

Panis, M. (2009). The Cultural Heritage and the New Tourist Territorialities in Rural Communities: an Emergent Reality in the Rincão Da Cruz District-City of Pelotas/RS. RA'E GA. O Espacio Geografico em Analise, 17, 77-92.

Pearce, D. (198I). Tourist Development. Londres: Longmans.

PhPclasses (2012). About Czech Republic. Recuperado el 3i de marzo de 20I4, de http://www.phpclasses.org/browse/country/cz/

Porto, S. M., Leanza, P. M. y Cascone, G. (2012). Developing Interpretation Plans to Promote Traditional Rural Buildings as Built Heritage Attractions. International Journal of Tourism Research, I4(5), 42I-436.

Skoczek, M. (2003). Rural Tourism as a Factor of Cultural Heritage Protection in Spain and Portugal. Prace i Studia Geograficzne, 32, 35-48.

Ziakas, V. y Costa, C. A. (2010). Between Theatre and Sport in a Rural Event: Evolving Unity and Community Development from the Inside-Out. Journal of Sport and Tourism, $15(\mathrm{I}), 7-26$. 